\title{
Thirty Meter Telescope Science Instruments: A Status Report
}

\author{
Luc Simard ${ }^{\mathrm{a}, \mathrm{b}}$, Brent Ellerbroek ${ }^{\mathrm{a}}$, Ravinder Bhatia ${ }^{\mathrm{a}}$, Matthew Radovan ${ }^{\mathrm{a}, \mathrm{c}}$, Eric Chisholm ${ }^{\mathrm{a}}$ \\ ${ }^{a}$ Thirty Meter Telescope Observatory, 100 West Walnut, Suite 300, Pasadena, CA, USA 91124 \\ ${ }^{\mathrm{b}}$ National Research Council of Canada, Herzberg Astrophysics and Astronomy Programs, 5071 \\ West Saanich, Victoria, Canada V9E 2E7 \\ ${ }^{c}$ University of California Observatories, 1156 High Street, Santa Cruz, USA 95065
}

\begin{abstract}
An overview of the current status of the science instruments for the Thirty Meter Telescope is presented. Three first-light instruments as well as a science calibration unit for AO-assisted instruments are under development. Developing instrument collaborations that can design and build these challenging instruments remains an area of intense activity. In addition to the instruments themselves, a preliminary design for a facility cryogenic cooling system based on gaseous helium turbine expanders has been completed. This system can deliver a total of 2.4 kilowatts of cooling power at $65 \mathrm{~K}$ to the instruments with essentially no vibrations. Finally, the process for developing future instruments beyond first light has been extensively discussed and will get under way in early 2017 .
\end{abstract}

\section{INTRODUCTION}

The first-light instrumentation suite for TMT includes the Narrow-Field InfraRed Adaptive Optics System (NFIRAOS), the Wide-Field Optical Spectrometer (WFOS), the InfraRed Imaging Spectrometer (IRIS), the InfraRed Multi-Slit Spectrometer (IRMS) and the NFIRAOS Science Calibration Unit (NSCU). NFIRAOS is described elsewhere in these proceedings ${ }^{1,2}$. This suite is supported by a set of observatory services including cryogenic cooling.

Good progress has been made over the last few years on all of the above. WFOS, a seeing-limited multi-object spectrometer, went through a "mini-studies" phase in which twelve teams looked at all of its subsystems to develop designs further. WFOS is now entering an opto-mechanical design and requirements phase that will focus on the end-toend optical design and key mechanical structure trade-offs. IRIS, an AO-assisted imager and integral field unit, is nearing its Preliminary Design Review. IRIS now features a 34" $\times 34^{\prime \prime}$ imager that makes full use of the NFIRAOS field-of-view with the best AO corrections. In order to minimize mass, parts of the IRIS structure now use carbon-fiber, and considerable attention has been given to its science cryostat design. The InfraRed Multi-slit Spectrometer (IRMS), a modified version of the Keck/MOSFIRE instrument, will go through a "mini-studies" phase that will look at end-to-end performance, fabrication and assembly and guider re-design. Work on the NSCU is resuming after a long hiatus. A oneyear preliminary design phase is getting underway with a new design, schedule and cost estimates being the main deliverables. The facility cryogenic cooling system has just completed a conceptual design phase in November 2015. This innovative system, which is based on gaseous helium turbine expanders, can deliver the required cooling power to all of the instruments with a negligible amount of vibration.

\section{INTERNATIONAL TEAM DEVELOPMENT}

TMT needs strong teams to deliver its challenging instruments and adaptive optics systems. There is strong interest from all partners in participating in instrument projects, and this interest is primarily driven by the interests of their respective science communities. TMT partners are separated by large geographical distances in addition to being used to different development models supported by a broad range of facilities and capabilities. It is therefore important that significant efforts be deployed to fully realize the exciting potential found within the partnership. Instrument teams must be built upon sensible scientific and technical collaborations while satisfying partner aspirations. TMT took a number of steps to achieve this goal. It started with planning and technical workshops. The goal of the planning workshops was to focus on processes and practices used in the development of TMT instruments and to put instrument teams in a strong position to effectively engage in TMT instrument efforts. They were intended for instrument builders as well as scientists interested in serving on instrument science teams. The topics relevant for scientists included how TMT science information is

Ground-based and Airborne Instrumentation for Astronomy VI, edited by Christopher J. Evans, Luc Simard, Hideki Takami Proc. of SPIE Vol. 9908, 99081V · C 2016 SPIE · CCC code: 0277-786X/16/\$18 · doi: 10.1117/12.2234058 
organized, the elements of a good science case, science flowdown within TMT systems engineering, and the role of science team members. The topics relevant for instrument builders included interfaces, instrument concept selection, development phases, planning and management practices, reviews, procurement models, and cost/schedule estimating methodologies. The technical workshops focused on requirements, designs, cost and schedule estimates for each instrument. The next step were the expressions of interest (EOIs). All interested teams were invited to submit EOIs. EOIs were not formal proposals, but they described in some detail the following: (1) specific instrument workshare(s), (2) science interests, (3) demonstrated, relevant expertise, (4) availability of resources and (5) expected role (leading/major/collaborating/supporting/supplier). Coordination and joint collaborations between partners to foster the "self-assembly" of instrument teams were of course encouraged as part of this submission process. Following the submission of these EOIs, TMT defined mini-studies that were initiated through a face-to-face "stakeholder" meeting. The word "mini" refers here to their short duration ( $\sim 1$ year) and limited budget ( $\$ 50-100 \mathrm{k})$. Each mini-study was reviewed and evaluated by a panel of TMT staff and appropriate independent experts.

Team building efforts through the above steps have been in full swing since 2014 and have resulted in many new additions to the TMT instrument building community. The latest TMT instrument world map is shown in Figure 1. A comparison between this map and the one published in these proceedings back in $2012^{3}$ will highlight the progress made on this important activity.

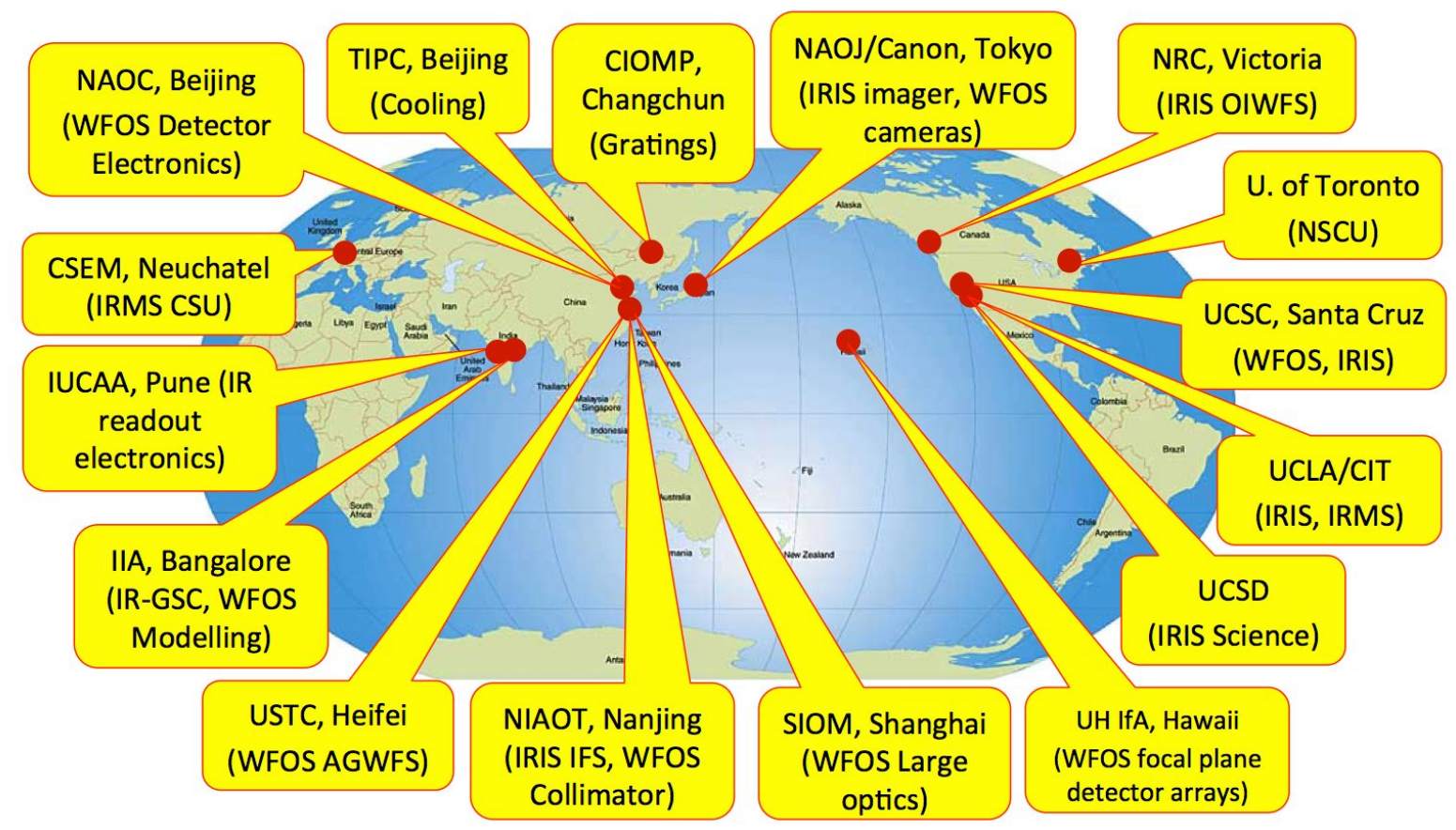

Figure 1. Teams involved in science instrument development across the TMT partnership.

\section{PROGRESS ON FIRST-LIGHT INSTRUMENT DESIGNS}

Figure 2 shows the first light instruments on the telescope. They are mounted on two Nasmyth platforms that provide long-term stability. The platforms are also easily re-configurable and large enough to support 8-10 instruments and their associated systems. The optical axis of TMT is 23 meters above the dome floor, and the Nasmyth platforms are 7 meters below this axis. The NSCU is mounted at the front of the NFIRAOS. IRIS and IRMS will be mounted on the bottom and top ports of NFIRAOS respectively, and the side port will not be populated at first light. This section describes progress made on the first-light instruments. 


\section{Wide-Field Optical Spectrometer}

WFOS, the Wide-Field Optical Spectrometer, is a seeing-limited, wide-field imaging multi-object spectrometer, and it was selected by the TMT Science Advisory Committee to be one of the first-light instruments for TMT. The history of WFOS stretches from 2005 to the present, and a number of concepts were studied throughout this period. The latest one was MOBIE, the Multi-Object Broadband Imaging Echellette ${ }^{4}$. MOBIE could provide direct imaging or create low $(\mathrm{R}=$ $\sim 1000$ ) to medium-resolution ( $\mathrm{R}=\sim 8,000)$ spectra in two color channels simultaneously, spanning $310-550 \mathrm{~nm}$ and 500 $1100 \mathrm{~nm}$ respectively. The rectangular field of MOBIE was approximately 3.0 arcmin x 8.3 arcmin resulting in a field area of 24.9 square arcminutes, and a total slit length of 500 arcsec. The MOBIE Feasibility Study Phase ran from June to December 2008, ending with a formal review by an external panel of experts. The Conceptual Design Phase was conducted in two stages due to funding limitations. The first stage ran from June 2009 to December 2010, after which a Value Engineering Study was conducted from January 2011 to February 2013. The second stage of the Conceptual Design ran from March 2012 to October 2013, concluding with a Handover Workshop. This workshop was not a formal review, and WFOS is therefore still formally at the conceptual design stage.

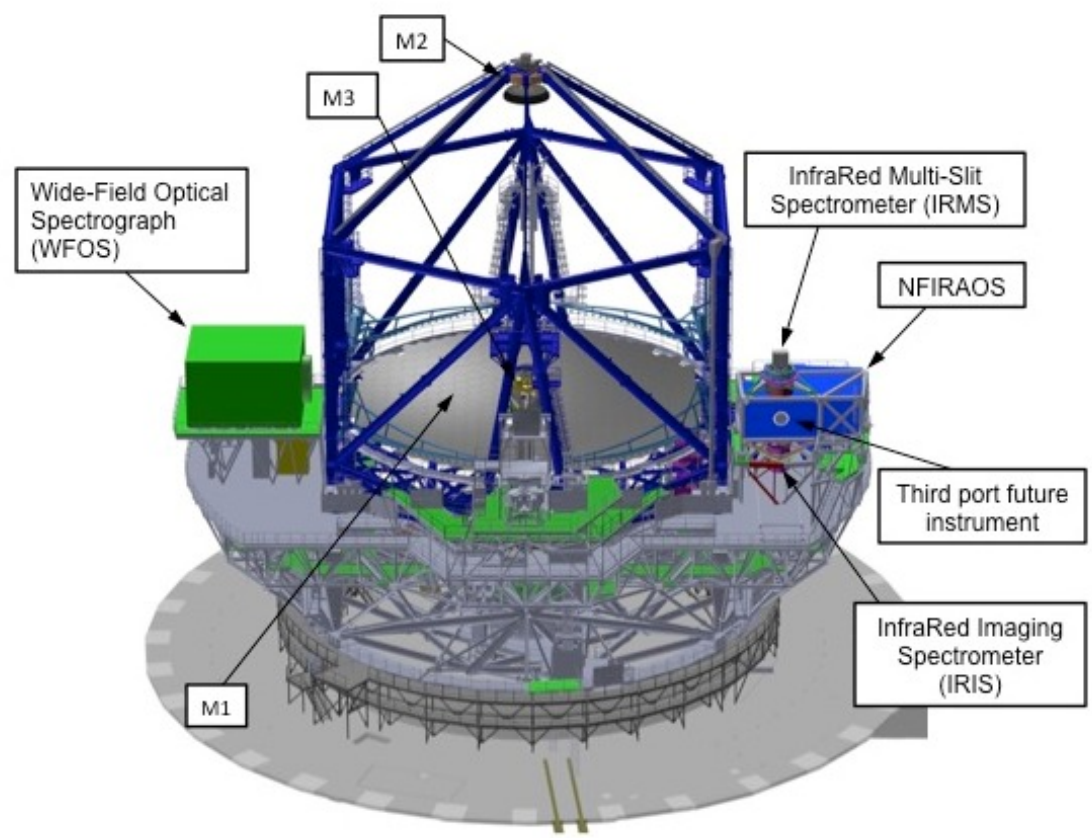

Figure 2. First-light instrumentation configuration on telescope.

Starting in October 2013, the TMT partnership has been engaged in an effort to form a full WFOS team through an international workshare development process. Mini-studies are a key element of this process, and their goals reached beyond technical work. Teams had the opportunity to: 1) review, understand and recommend revisions to the WFOS requirements, 2) identify problems/inconsistencies/issues and provide creative solutions to resolve them, 3) build and establish new institution relationships as work coordination at multiple participating institutions was developed and optimized, 4) demonstrate an understanding of TMT technical and management requirements for deliverables and reporting, and 5) demonstrate relevant technical expertise. By the end of the mini-study phase, all participating institutions had been engaged in meaningful and challenging WFOS technical work and were familiar with TMT standard project management processes.

Twenty-one expressions of interest (EOIs) were submitted to TMT by various groups in the United States, China, India and Japan. These EOIs provided an invaluable source of information on relevant expertise, past experience, human resources and facilities. The potential for instrumentation work across the TMT partnership is most impressive not only 
for WFOS but also future instruments. Based on the submitted EOIs, the TMT instrumentation group drafted a list of 12 mini-studies (see Table 1). This list was driven by the following considerations: (1) expressed interests and relevant expertise, (2) areas of the current WFOS design that would benefit from some technical work as identified in the MOBIE CDP Handover Workshop panel report or as required by other ongoing TMT project-level design work, (3) maintaining sensible and well-defined interfaces between subsystem work at different locations and (4) exploring promising collaborations across TMT partners.

Table 1. List of 2015 WFOS Mini-Studies

\begin{tabular}{|c|c|c|}
\hline Description & $\begin{array}{c}\text { Managing } \\
\text { Institution }\end{array}$ & Participating Institutions \\
\hline $\begin{array}{c}\text { Mask Exchanger System Trade-off and Fabrication } \\
\text { (MEX) }\end{array}$ & USTC & UCO, NAOJ, ASIAA, USTC \\
\hline AGWFS and Operational Concepts (WGS) & USTC & IIA, IUCAA, NIAOT,USTC \\
\hline Instrument Modelling (SYS) & UCO & IIA, SJTU, UCO \\
\hline Off-Axis Collimator Mirror (COL) & NIAOT & NIAOT \\
\hline Atmospheric Dispersion Corrector (ADC) & USTC & NAOT, XMU, USTC \\
\hline $\begin{array}{c}\text { Metrology, Calibrations and Instrument Simulations } \\
\text { (MCS) }\end{array}$ & UCO CIT \\
\hline $\begin{array}{c}\text { Spectrometer Camera Systems (CAM) } \\
\text { Folding Optical Systems (FOS) }\end{array}$ & NAOJ & SIOM \\
\hline Grating Exchange System (GEX) & SIOM & XMU \\
\hline End-to-end Optical Design and Stray Light Analysis \\
(OPT/STL)
\end{tabular}

Unfortunately, it was not possible (nor realistic) to attach a mini-study to every submitted EOI. The prime concern here was keeping the overall mini-study effort manageable, and twelve mini-studies was deemed to remain sensible. Launching all these mini-studies, keeping them on track through completion and reviewing them was a challenge all in itself. The mini-studies were formally launched at a meeting at the National Astronomical Observatories of China (NAOC) in Beijing in April 2014 and underwent a formal review by an external panel of experts in April 2015. Each mini-study had a local manager. Teams typically met bi-weekly, and all managers met bi-weekly as well. Two sets of interim reviews were also held over the course of that year. Fifteen institutes in four countries were involved. They were the Academia Sinica Institute of Astronomy and Astrophysics (ASIAA), Aryabhatta Research Institute of Observational Sciences (ARIES), California Institute of Technology (CIT), Changchun Institute of Optics, Fine Mechanics and Physics (CIOMP), Indian Institute of Astrophysics (IIA), Inter-University Center for Astronomy and Astrophysics (IUCAA), Nanjing Institute of Astronomical Optics and Technology (NIAOT), National Astronomical Observatories of China (NAOC), National Astronomical Observatory of Japan (NAOJ), Shanghai Institute of Optics and Fine Mechanics (SIOM), Shanghai Jiao Tong University (SJTU), University of California Observatories (UCO), University of Hawaii (UH), University of Science and Technology of China (USTC), and Xiamen University (XMU).

The mini-studies yielded a wealth of new technical information and new ideas on mask and grating exchange systems, very large gratings, optical distortion mapping, seismic response, camera design and buildability and detector focal plane arrays to name a few. The outcomes of the mini-studies are described elsewhere in these proceedings ${ }^{5,6,7}$. As an example, Figure 3 shows new concepts for the mask and grating exchange systems based on assembly-line robots. The WFOS multi-object masks are large $(1100 \mathrm{~mm} \times 700 \mathrm{~mm})$, and the minimum number of masks required per night was initially 
ten. A stack of ten masks is therefore a sizable assembly, and this assembly was located on-board the instrument rotating structure. Moving the whole assembly off this structure would remove the space limit on the number of masks. The masks would then simply be loaded into the instrument by the robot as required by the observing programs. One of the Chinese institutes involved in the mini-studies had such a robot in their laboratory and was therefore able to confirm that mask exchanges could be safely done in this way within the time limit in the WFOS target acquisition time budget ${ }^{7}$. Figure 3 also shows a similar approach for the grating exchange system. The grating drum assemblies are $0.5-\mathrm{m}$ in diameter, and there are multiple gratings in each spectral channel. Removing them from the rotating structure would allow the design team to decrease the diameter and mass of the rotating structure. These robotic configuration options will be studied in more details in the next phases of the WFOS development.
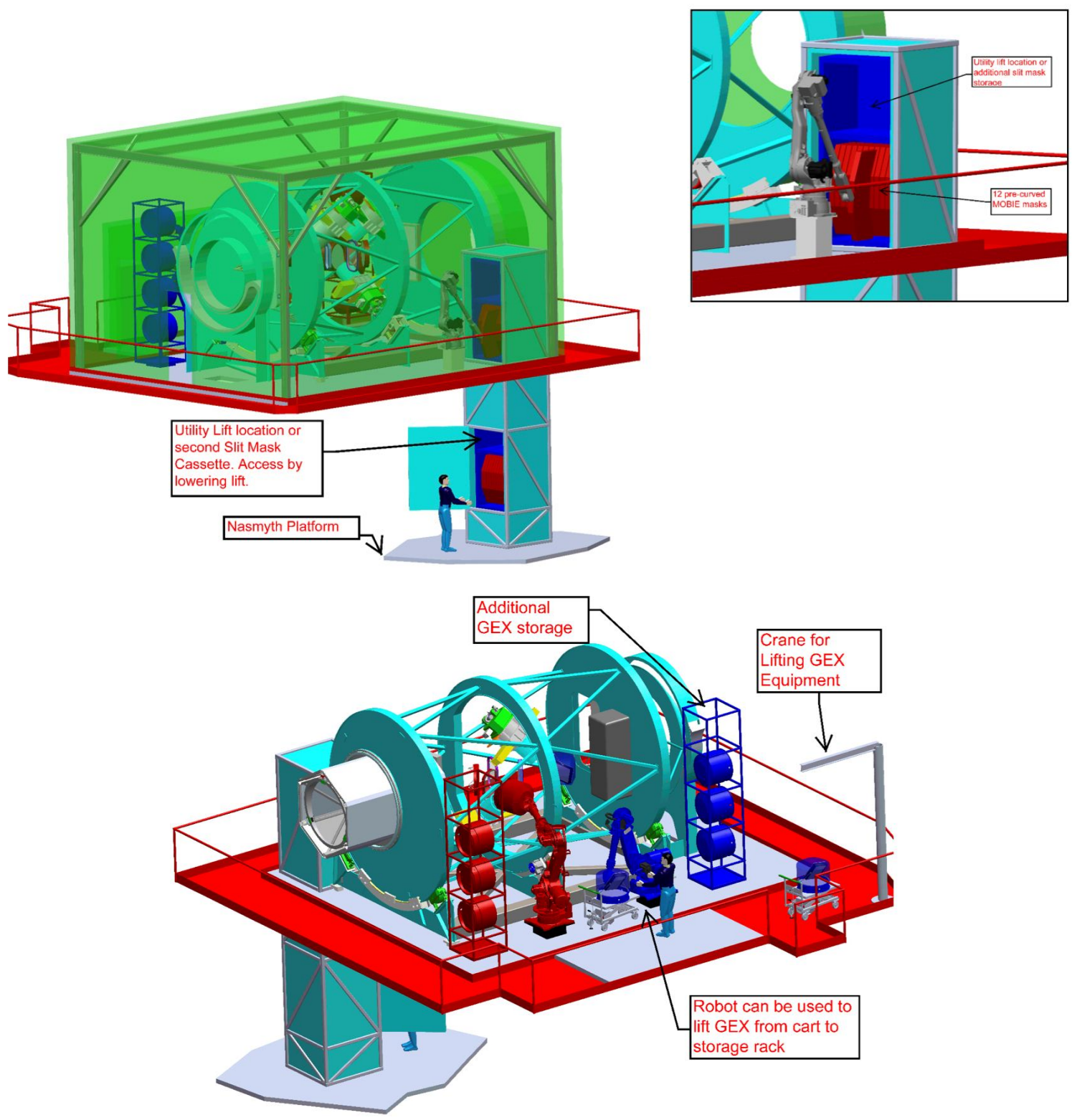

Figure 3. (Top) WFOS mask exchange system based on an assembly-line robot. The masks are brought up to the robot in an elevator. (Bottom) Similar approach for the grating exchange system on the opposite side of the instrument. 
The next phase is known as the "Opto-Mechanical Design and Requirements" phase, and it will focus on the end-to-end optical design and key mechanical trade-offs. It started in January 2016, and it is expected to take one year.

Previous design work for WFOS-MOBIE relied on an Echellette spectrometer concept that achieved beam clearance with a large (nearly $20^{\circ}$ ) out-of plane (quasi-Littrow) angle on the gratings. Subsequent analysis of the system distortion and sensitivity to flexure from the MCS mini-study revealed large, uncorrectable spectral tilt and complicated distortion behavior which proved impossible to correct with flexure compensation systems implemented thus far in previous instruments. An initial study of alternate grating architectures indicated that this behavior could be improved with a trade between the quasi-Littrow configuration and a greater in-plane angle. Initial work presented in the 2015 OPT/STL ministudy final report was promising enough to warrant further study. As part of the OMDR work, UCO will continue to work with IIA to compare different dispersive element architectures, including varying the in-plane/out-of-plane angles as well as the cross-dispersing prism apex, incidence, and rotation angles. Realistic, buildable systems will be developed and analyzed for each of the three spectral resolution modes in both the red and blue channels. An attempt will also be made to quantify the effects of the collimator and camera performance on the above described distortion and sensitivity that will contribute to the development of the error budget allotted to these optical systems.

Given that the WFOS structure is not gravity invariant, instrument flexure, if not properly controlled, could limit scientific productivity. The WFOS MCS mini-study team discovered that the optical design has unacceptable distortion sensitivity for motion estimates that were the product of simple scaling laws and measured 10-meter class multi-object spectrometer flexure performance. For WFOS to be viable, not only the optical design must be changed to reduce the distortion sensitivity, but the structural design must also be optimized to have better flexure performance than any 8 to 10 meter class multi-object spectrometers ever built.

Previous design work for the spectrometer cameras for the WFOS-MOBIE design concentrated on all refractive solutions that may have potential manufacturability issues. The polishing of aspheric refractive surfaces on the preferred optical materials is very challenging, though progress is being made within the WFOS project to address these issues. Still, the cost of complex refractive systems and their associated schedule uncertainties calls for a prudent consideration of alternative designs. In particular, although catadioptric designs have only been given an initial look, they show some promise, particularly when care is taken to maximize optical transmission. This work to be undertaken at CIT will develop a hybrid Matsukov catadioptric camera subsystem design that optimizes, as a system, the optics and CCD cryostat lying on-axis but inducing minimal optical obscuration. CIT has recently engaged in significant engineering of a minimal-obscuration cryostat and wide-field optics for the Zwicky Transient Facility (ZTF). It is envisioned that these conceptual principles will be followed to evaluate the logical end-point of catadioptric designs and enable a technical and cost/risk comparison with all-refractive designs under development by other partners within the WFOS consortium.

\section{InfraRed Imaging Spectrometer}

The IRIS team has made significant progress towards its Preliminary Design Review slated for November 2016. More details are presented in many other contributions to these proceedings $8,9,10,11,12,13,14,15,16$, and only a very brief summary is given here. The baseline is now the "Sequential Design" which has a number of advantages over previous designs: (1) Reduced number of optical elements, (2) natural expansion of the imaging field of view from 17" to 34", and (3) modularity of the science cryostat. The first two advantages come from the fact that the imager now acts as the reimaging optics for the spectrometer, and a number of fore-optic elements were thus eliminated from the design. The larger field of view will be critical to a number of science cases and will make full use of the NFIRAOS field of view with the best level of AO corrections $\left(30^{\prime \prime}\right)$. Recent high-fidelity simulated images produced by the IRIS science team ${ }^{17,18}$ have shown the exciting potential of this enhanced imaging capability in a spectacular way.

Progress has been made on all subsystems including a new modular science cryostat design (Figure 4). The top section of the cryostat contains the imager. The imager is under the responsibility of the National Astronomical Observatory of Japan (NAOJ) in Mitaka, Japan. This section of the cryostat can operate as a self-contained cryogenic environment in which the imager will be fully tested after integration at NAOJ and prior to shipping to the IRIS lead integration site in California. The bottom section contains the lenslet-based and slicer-based IFU spectrometers. These IFUs are being 
designed and built at UCLA and CIT respectively, and they will also need to be tested in a self-contained way prior to integration with the other subsystems.
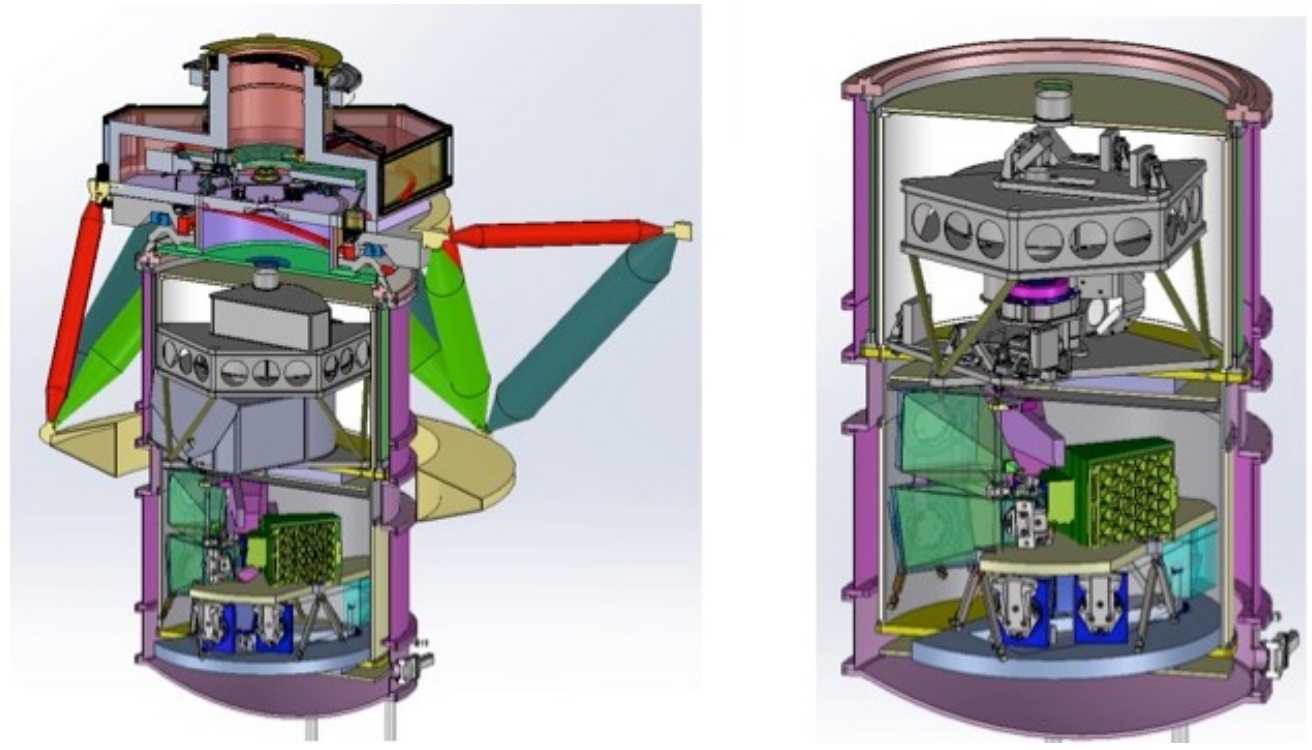

Figure 4. The latest IRIS mechanical design. (Left) Full instrument assembly including the On-Instrument Wavefront Sensor module, the science cryostat and the rotator and mounting struts. (Right) The IRIS science cryostat itself showing the modularity of the design.

In order to meet the IRIS total mass requirement, the team has spent considerable efforts on light-weighting the support structure connecting IRIS to NFIRAOS, and steel was replaced by carbon fiber ${ }^{12}$.

The main milestones in the current working schedule for IRIS are fabrication and assembly starting in early 2019 , integration starting in mid-2021, delivery at the NFIRAOS integration site in 2022 and ready to be shipped to the TMT site in 2024.

\section{InfraRed Multi-Slit Spectrometer (IRMS)}

IRMS, the InfraRed Multi-slit Spectrometer, is an AO-assisted, near-IR, multi-object spectrometer for TMT. IRMS is a modified version of the MOSFIRE instrument at Keck and now in full science use. Although MOSFIRE is a seeinglimited instrument for Keck, it can be adapted for use in an AO mode with NFIRAOS, providing a powerful capability for TMT at low risk and modest cost. When optimized for wide-field mode, NFIRAOS will deliver images to IRMS that will produce almost an order of magnitude gain in encircled energy within narrow (160 mas) slits over the entire $2^{\prime}$ diameter field of view. MOSFIRE is designed for the $\mathrm{f} / 15$ Cassegrain focus on the Keck 1 telescope. This is the same $\mathrm{f} /$ ratio as for the NFIRAOS output port and without change to the MOSFIRE design, it would naturally take in the entirety of the NFIRAOS $2^{\prime}$-diameter field of regard. It will cover the wavelength range $0.95 \mu \mathrm{m}$ to $2.45 \mu \mathrm{m}$ with spectral resolution of up to $R=4660$ for a $0^{\prime \prime} .16$ slit. A configurable slit mask unit allows on-the-fly, slitlet reconfigurations without the need to swap masks in and out of its cryogenic environment.

A modest IRMS “mini-study" was conducted at CIT from August 2011 through April 2013. The primary goals of this mini-study was to confirm that the MOSFIRE optical design could be modified to work with the NFIRAOS output optical prescription, develop a design for the NFIRAOS interface (rotator and cable wrap), to design the IRMS OnInstrument Wavefront Sensor subsystem and to provide cost and schedule estimates. The mini-study demonstrated the viability of using the MOSFIRE design for IRMS. 
The TMT partnership is now engaged in an effort to form a full IRMS team through an international workshare development process similar to the one used for WFOS. The next step is to initiate a set of three mini-studies in Fall 2016: (1) Detailed performance modelling including the benefits of AO and using IRMS on a 8-m class telescope in the interim, (2) Fabrication and assembly demonstration and (3) On-Instrument Wavefront Sensor subsystem (re-)design.

The main difference between Keck/MOSFIRE and TMT/IRMS is that the latter will be fed by an AO system namely NFIRAOS. The main performance metric is the fraction of light going into an IRMS slitlet. The use of an AO feed is expected to increase this fraction significantly. There have also been discussions on building IRMS right now and using it on an existing $8-10 \mathrm{~m}$ class telescope until TMT is ready to receive it. In this case, the optical prescription of IRMS would not be an ideal match to the telescope optics, and a degradation in performance would follow. Still, this option would mitigate many risks related to IRMS, and a reasonable degradation would be acceptable. This mini-study will be tightly coupled to the IRMS science cases and operational concepts and will include the following tasks: (1) model the fraction of light going into a IRMS slitlet as a function of position within the IRMS field of view using realistic NFIRAOS point-spread-functions, (2) model the performance of IRMS in its seeing-limited mode under which the NFIRAOS deformable mirrors are held in a static state, and (3) model the performance of IRMS operating on an existing 8-10m telescope.

It is expected that a significant fraction of IRMS will be fabricated, assembled and tested at TMT partner institutions that were not involved in MOSFIRE. The goal of this mini-study is therefore for these new institutions to demonstrate that they can leverage their expertise in cryogenic instrumentation to provide all required IRMS elements.

The On-Instrument Wavefront Sensor (OIWFS) subsystem is one area of the MOSFIRE design that is expected to undergo significant changes to adapt IRMS to the NFIRAOS interface. It is vitally important to re-design this subsystem as soon as possible to estimate the extent and impact of this re-design on the cost and schedule for IRMS. This design work will establish the optimal OIWFS patrol strategy based on IRMS science cases, observing scenarios and guide star availability, develop the mechanical design of the OIWFS patrol assembly and assess its impact on the NFIRAOS-IRMS interface, develop an OIWFS opto-mechanical design with the goal of re-using as much of the IRIS OIWFS design as possible and assess/characterize various detector array options in terms of their field-of-view, flux sensitivity, frame rate, dark current and readout noise.

\section{NFIRAOS Science Calibration Unit}

The NFIRAOS Science Calibration Unit (NSCU) will provide all required calibrations for the instruments fed by NFIRAOS. It must therefore support a fairly wide range of AO-assisted capabilities: high-spatial resolution imaging, multi-slit spectroscopy, integral-field spectroscopy and high spectral resolution spectroscopy. The unit will be deployed in front of NFIRAOS to ensure that the calibration light path reproduces the science light path as much as possible. The calibrations fall into three types: flat-fielding, wavelength and pupil masking. The first two are typically found in calibration units. The novelty here will come from the choice of light sources. There have been significant advances in the design and implementation of new kinds of sources such as super-continuum sources for flat-fields and laser combs for wavelength calibrations. These advances will be considered in the NSCU design. The third type (pupil masking) is specific to the requirements of NFIRAOS. The NSCU will feature a rotating pupil mask at the output port of its integrating sphere to simulate the illumination of the telescope onto NFIRAOS, its client instruments and their wavefront sensors. The chief motivation is to provide a position and shape reference to enable calibrating the pupil pointing lookup tables for the telescope via a truth wavefront sensor in NFIRAOS. The pupil mask will imitate the TMT pupil including major spider structures, secondary mirror shadow and the primary mirror serrated edge.

The NSCU is a Canadian workshare deliverable. Its design and construction will be led by the University of Toronto in collaboration with Canadian industrial partners. Early designs were studied in 2009-2010 ${ }^{19}$, but work on the NSCU has been on hiatus since then. It is now resuming with plans for a one-year preliminary design phase followed by a fabrication and assembly phase. The NSCU should be ready to be mounted on NFIRAOS in 2020. 


\section{FACILITY CRYOGENIC COOLING SYSTEM}

A facility cryogenic cooling system for the instruments including the Alignment and Phasing System (APS) has a number of attractive benefits: (1) it turns cooling into another observatory-level service like electrical power, (2) sources of vibration can be controlled and minimized, (3) redundancy can be built-in and (4) it can be designed to be scalable and grow with the instrument suite. Vibration is of particular concern as it has negatively impacted the performance of adaptive optics systems on existing $8-10 \mathrm{~m}$ telescopes. Cryocoolers are significant sources of vibrations, and detailed modelling against the TMT AO performance vibration budget ${ }^{20}$ indicates that even the quietest commercial cryocoolers may not be sufficient to meet allowed vibration levels. A new solution therefore needed to be explored.

The cryogenic cooling group at the Technical Institute of Physics and Chemistry (TIPC) of the Chinese Academy of Sciences has taken a novel design approach based on reverse-Brayton cycle turbine expanders that delivers long operating lifetimes, a wide temperature range, a large cooling capability and, most importantly, very low levels of vibration $^{21}$. The basic layout of this system is shown in Figure 5. The system is scalable and can be implemented to only support first-light instruments at the beginning and the full suite of planned instruments later on. The full system consists of two warm compressors located in the TMT summit facility building. Gaseous helium at ambient temperature is then sent from these compressors through the telescope structure azimuth wrap to two cold boxes located on the Nasmyth platforms (total distance: 125 meters). The cold boxes contain turbine expanders that cool the gas down to $65 \mathrm{~K}$. The cold gas is then routed to individual instruments through three cryogenic valve boxes connected to vacuum-jacketed lines.

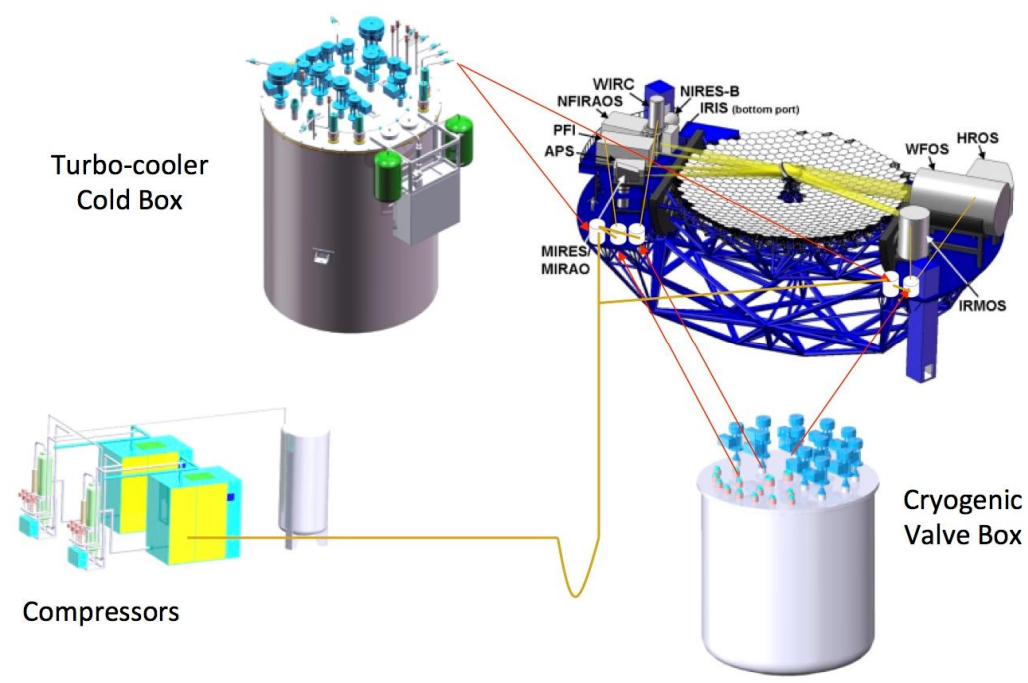

Figure 5. Schematic layout of the facility cooling system with turbo-cooler cold boxes and cryogenic valve boxes. The vibrationally noisy compressors are located in the summit facility building far away from the instruments on the Nasmyth platforms.

The TIPC conceptual design, which produces $2.9 \mathrm{~kW}$ of cooling power at $65 \mathrm{~K}$ and $0.4 \mathrm{~kW}$ at $160 \mathrm{~K}$, passed a formal TMT external review in November 2015. Work is now focussed on refining instrument cooling requirements and exploring further system design trade-offs.

\section{PLANNING FUTURE INSTRUMENTS}

The development of new, powerful instruments is obviously essential to maintaining TMT's scientific effectiveness. It is driven by the recognition that capabilities similar to the proposed planned instrument suite are required to fulfill TMT science mission, and that these capabilities will be needed as soon as possible after first light. The goal of the instrument development program is to maintain TMT at the forefront of international astronomical research over the expected 
lifetime of the Observatory. Every TMT instrumentation project will involve a very sizable investment of resources, and will have to be undertaken by a consortium of institutions. Recent instrumentation projects on $8-10 \mathrm{~m}$ class telescopes (e.g., VLT/MUSE, LBT/LINC-NIRVANA, Gemini/GPI, Keck/MOSFIRE) are perfect examples of this. Instrumentation team participants can come from TMT partners as well as from institutions from the broader community.

The future instrumentation development program for TMT has been defined over the last five years through the discussions with the instrument building community, the Scientific Advisory Committee (SAC) and the Board. The process starts with "community explorations". This is where new instrumentation ideas for TMT are born. They are meant to inform the prioritization of desired TMT instrumentation capabilities by the SAC. Even though not all community explorations may be initiated by SAC, SAC will play a central role in coordinating them through the TMT Instrumentation Development Office (IDO). Community explorations will be used by SAC to draft initial science requirements (and their rationale) at a level of detail sufficient enough to avoid misunderstanding and potentially wasted efforts. Given that both the scientific and technical landscapes can change significantly over time, it is important to survey both on relatively short timescales ( 1 year). Explorations are therefore expected to provide a constant stream of information to SAC even though calls for more advanced development phases may be issued at less frequent intervals ( 2-3 years). Community explorations will be open to unsolicited proposals and new technological development ideas. Explorations will also guide SAC in suggesting a cost cap for a given scientific capability to ensure that instrument teams are given clear cost guidance from the start. There will be at least three types of community explorations: consultations (e.g., white papers, workshops, etc.), technology development testbeds (e.g., coronographs, wavefront sensors, control algorithms) and full feasibility studies.

The next step will be the establishment of priorities by the SAC. This is the cornerstone of the TMT instrumentation program. SAC is responsible for maintaining the list of TMT instrumentation priorities. SAC will update this list on a regular basis based on the outcomes of the community explorations. Regarding the optimal rate of arrival of secondgeneration instruments, SAC has agreed that the minimum acceptable rate would be an average of one instrument every three years, and that the goal should be one instrument every two years. Based on this recommendation, SAC will update its instrumentation priorities on a similar timescale to coincide with Calls for Proposals for new instruments. Instrumentation selection must be primarily science-driven, but it must also factor in all available information on technical readiness, schedule, cost and the overall mix of commissioned and planned instrumentation at TMT.

Very-well defined requirements will be essential. In addition to the "numbers" themselves, the rationale behind the requirements will be very useful so that it will be clearer what parts might be negotiable if cost/risk become excessive. This is the approach that SAC followed for the selection of the TMT first-light instruments in 2006, and the success of this process will be used as a template for the future. It is recognized that there are two broad classes of instrument capabilities: workhorse versus "experiment-driven". The focus of a workhorse capability is to open up a wide region of parameter space in response to a broad community interest whereas an "experiment-driven" capability will purse a more focused science goal with a particularly high science impact. SAC will apply the guiding principle that workhorse capabilities should generally be implemented first in considering the relative priorities of these two classes of instruments. It should be re-emphasized that the SAC prioritization process includes both AO systems and science instruments. Given the tight integration between $\mathrm{AO}$ and instrument systems, this may look like a superficial distinction at first glance, but there will be cases where they will have to be upgraded/replaced on different timescales. Prioritization metrics will be applied consistently to both sets of systems as much as possible with the realization that technical readiness is a particularly important criterion for AO systems. A Call for Proposals for conceptual design level studies will normally be issued every three years. It is expected that two instrument concepts will be studied per cycle with two studies per concept. Proposals received by the Observatory will be reviewed by external panels that will provide advice to the IDO, SAC and Board as to which proposals to pursue.

One interesting consideration regarding future instrumentation choices will be the complementarity between instrument suites on all ELTs. Table 2 shows the equivalence between the GMT, TMT and E-ELT suites ${ }^{22,23}$. This is not a one-toone mapping, but it does show how they overlap and differ. There are striking differences between all three. First, a near-infrared, AO-assisted imager/IFU instrument will be available at all three telescopes, but the similarity stops here. Both GMT and TMT will have a first-light multi-object spectrometer, but E-ELT will not. GMT will have the only firstlight, high-resolution spectrometer (G-CLEF), and E-ELT will be the only one with a mid-infrared capability (METIS). E-ELT will also have the diffraction-limited imager with the largest field of view (MICADO). These differences at first light may be surprising, but they can be understood given the wide range of possible science objectives and the 
fundamentally different telescope design choices made by these projects. It remains an open question whether such differences will persist in future instrument generations, but the complexity and cost of ELT-class instruments will certainly lead to joint coordination to avoid duplication wherever possible and still benefit all communities involved.

The next step in the development of future instruments for TMT is expected to be a call for feasibility study proposals in early 2017. These feasibility studies would then be completed and externally reviewed in early 2019 , and very detailed conceptual design studies would be launched in 2020.

Table 2. ELT Instrumentation Equivalence Table. First-light instruments are underlined.

\begin{tabular}{|l|c|c|c|}
\hline \multicolumn{1}{|c|}{ Type of Instrument } & GMT & TMT & E-ELT \\
\hline Near-IR, AO-assisted Imager + IFU & $\underline{\text { GMTIFS }}$ & $\underline{\underline{\text { IRIS }}}$ & $\underline{\underline{\text { HARMONI }}}$ \\
\hline $\begin{array}{l}\text { Wide-Field, Optical Multi-Object } \\
\text { Spectrometer }\end{array}$ & $\underline{\text { GMACS }}$ & $\underline{\text { WFOS }}$ & $\begin{array}{c}\text { MOSAIC- } \\
\text { HMM }\end{array}$ \\
\hline Near-IR Multislit Spectrometer & NIRMOS & $\underline{\text { IRMS }}$ & $\begin{array}{c}\text { MOSAIC- } \\
\text { HMM }\end{array}$ \\
\hline $\begin{array}{l}\text { Deployable, Multi-IFU Imaging } \\
\text { Spectrometer }\end{array}$ & & IRMOS & $\begin{array}{c}\text { MOSAIC- } \\
\text { HDM }\end{array}$ \\
\hline $\begin{array}{l}\text { Mid-IR, AO-assisted Echelle } \\
\text { Spectrometer }\end{array}$ & TIGER & PFI & ELT-PCS \\
\hline High-Contrast Exoplanet Imager & GMTNIRS & NIRES & HIRES \\
\hline $\begin{array}{l}\text { Near-IR, AO-assisted Echelle } \\
\text { Spectrometer }\end{array}$ & $\underline{\text { G-CLEF }}$ & HROS & HIRES \\
\hline High-Resolution Optical Spectrometer & WIRC & $\underline{\text { MICADO }}$ \\
\hline "Wide"-Field AO-assisted Imager & & & \\
\hline
\end{tabular}

\section{ACKNOWLEDGEMENTS}

The TMT Project gratefully acknowledges the support of the TMT collaborating institutions. They are the California Institute of Technology, the University of California, the National Astronomical Observatory of Japan, the National Astronomical Observatories of China and their consortium partners, the Department of Science and Technology of India and their supported institutes, and the National Research Council of Canada. This work was supported as well by the Gordon and Betty Moore Foundation, the Canada Foundation for Innovation, the Ontario Ministry of Research and Innovation, the Natural Sciences and Engineering Research Council of Canada, the British Columbia Knowledge Development Fund, the Association of Canadian Universities for Research in Astronomy (ACURA), the Association of Universities for Research in Astronomy (AURA), the U.S. National Science Foundation, the National Institutes of Natural Sciences of Japan, and the Department of Atomic Energy of India.

\section{REFERENCES}

[1] Boyer, C. et al., “Adaptive optics program update at TMT,” Proc. SPIE 9909-08 (2016)

[2] Herriot, G. et al., "NFIRAOS AO for the Thirty Meter Telescope," Proc. SPIE 9909-102 (2016)

[3] Simard, L. et al., "The instrumentation program for the Thirty-Meter Telescope," Proc. SPIE 8446-49 (2012) 
[4] Bernstein, R. A. et al., "Progress of the conceptual design for the MOBIE imaging spectrograph for TMT," Proc. SPIE 7735-77 (2010)

[5] Radovan, M. et al., "The wide-field optical spectrometer for TMT," Proc. SPIE 9908-80 (2016)

[6] Ozaki, S. et al., "Polishing test of a poly-crystal calcium fluoride lens: toward the development of TMT WFOS," Proc. SPIE 9908-357 (2016)

[7] Zhang, F. et al., "Design and study of the mask exchange system based on Delta parallel mechanism," Proc. SPIE 9906-114 (2016)

[8] Larkin, J. E. et al., “The Infrared Imaging Spectrograph (IRIS) for TMT: instrument overview,” Proc. SPIE 9908-70 (2016)

[9] Zhang, K. et al., "The Infrared Imaging Spectrograph (IRIS) for TMT: preliminary design of image slicer," Proc. SPIE 9908-354 (2016)

[10] Phillips, A. C. et al., "The Infrared Imaging Spectrograph (IRIS) for TMT: the ADC optical design," Proc. SPIE 9908-373 (2016)

[11] Uraguchi, F. et al., "The Infrared Imaging Spectrograph (IRIS) for TMT: Prototyping of cryogenic compatible stage for the Imager," Proc. SPIE 9908-380 (2016)

[12] Dunn, J. S. et al., "The Infrared Imaging Spectrograph (IRIS) for TMT: multi-tiered wavefront measurements and novel mechanical design," Proc. SPIE 9908-381 (2016)

[13] Schöck, M. et al., "Flowdown of the TMT astrometry error budget(s) to the design of the first-light instrument IRIS,” Proc. SPIE 9908-385 (2016)

[14] Tsuzuki, T. et al., "The Infrared Imaging Spectrograph (IRIS) for TMT: optical design of IRIS imager with co-axis double TMA," Proc. SPIE 9908-386 (2016)

[15] Chapin, E. L. et al, "The Infrared Imaging Spectrograph (IRIS) for TMT: motion planning with collision avoidance for the on-instrument wavefront sensors," Proc. SPIE 9913-29 (2016)

[16] Weiss, J. L. et al., "The Infrared Imaging Spectrograph (IRIS) for TMT: software design,” Proc. SPIE 9913-104 (2016)

[17] Wright, S. A. et al., "The infrared imaging spectrograph (IRIS) for TMT: latest science cases and simulations," Proc. SPIE 9909-5 (2016)

[18] Walth, G. et al., "The Infrared Imaging Spectrograph (IRIS) for TMT: data reduction system,” Proc. SPIE 9913-165 (2016)

[19] Moon, D.-S. et al., "The science calibration system for the TMT NFIRAOS and client instruments: requirements and design studies," Proc. SPIE 7735-209 (2010)

[20] Thompson, H. A. et al., "Measurement of vibration source amplitudes including a low-vibration centralized cryocooling system against the TMT AO performance vibration budget." Proc. SPIE 9911-37 (2016)

[21] Peng, N. et al., "Conceptual design of the TMT instruments cryogenic cooling system," Proc. SPIE 9911-63 (2016)

[22] Jacoby, G. H. et al., "Instrumentation progress at the Giant Magellan Telescope project," Proc. SPIE 9908-68 (2016) SPIE 2016

[23] Casali, M. M. et al., "Progress along the E-ELT instrumentation roadmap," Proc. SPIE 9908-67 (2016) 